1 King's College London, Department of Child and Adolescent Psychiatry, Institute of Psychiatry, Psychology \& Neuroscience, London, UK

2 King's College London, Social, Genetic and Developmental Psychiatry Centre, Institute of Psychiatry, Psychology \& Neuroscience, London, UK

3 South London and Maudsley NHS Foundation Trust, National and Specialist CAMHS Clinic for Trauma, Anxiety, and Depression, London, UK

4 Department of Psychology, Harvard University, Cambridge MA, USA

5 Department of Psychology, Kingston University, London, UK

6 Yale Early Stress and Adversity Consortium, Yale University Child Study Center, New Haven CT, USA

Correspondence to: A Danese andrea.danese@kcl.ac.uk Cite this as: BMJ 2020;371:m3073 http://dx.doi.org/10.1136/bmj.m3073 Published: 19 November 2020

\title{
Psychopathology in children exposed to trauma: detection and intervention needed to reduce downstream burden
}

The clinical implementation of assessment and evidence based interventions is lagging behind research, with huge cost to individuals and society, write Andrea Danese and colleagues. To provide the best possible care to some of the most vulnerable children, specialist training, clinical capacity, and access to care must be increased

\section{Andrea Danese, ${ }^{1,2,3}$ Katie A McLaughlin, ${ }^{4}$ Muthanna Samara, ${ }^{5}$ Carla S Stover ${ }^{6}$}

Childhood traumas-defined as events that involve "actual or threatened death, serious injury or accident, or sexual violence"1-are key modifiable risk factors for psychopathology in children, adolescents, and adults. As such, child trauma is a major focus for research and clinical practice in mental health. ${ }^{2}$ Although much is known about the association of child trauma with psychopathology, ${ }^{3-11}$ and about evidence based interventions for trauma related psychopathology,,$^{12-14}$ the clinical implementation of adequate assessment and treatment in this area has been hampered by substantial obstacles in service delivery. For example, although some children are resilient in the face of trauma, many trauma-exposed children develop complex psychopathology that challenges diagnostic boundaries and simple case formulation. ${ }^{4}$ To identify and address the needs of this vulnerable group, we need to boost specialist training and clinical capacity in child trauma and its consequences.

Furthermore, although effective treatments exist, ${ }^{12-14}$ most children with trauma-related psychopathology probably go undetected and do not access treatment promptly. To tackle these unmet needs, it is important to identify barriers to healthcare use, implement screening procedures, develop accurate prediction models to detect children at most risk, and improve primary care practitioners' knowledge of child and adolescent mental health problems. Upfront investment to tackle these barriers to service delivery can reduce the large downstream costs to individual children and society.

\section{Mental ill health in children after trauma}

After traumatic events, most children show transient psychological symptoms. They may become tearful, anxious, clingy, or withdrawn; struggle to pay attention or to sleep; re-enact the event through play and drawings; avoid talking about it; complain about headaches or stomach aches; or become irritable and oppositional as they struggle to cope with their emotions. These are normal psychological responses to trauma that subside within a few weeks in most children. When psychological symptoms persist for more than a month and impair the child's functioning (such as school achievement or socialisation with peers), children may meet criteria for a psychiatric diagnosis.
A common clinical concern is post-traumatic stress disorder (PTSD). ${ }^{1}$ In PTSD, abnormal processing of traumatic memories leads to persistent

re-experiencing of the event through unwanted and distressing memories or nightmares, particularly in the presence of triggers resembling the context in which the traumatic event occurred. The memories typically are experienced as if they were happening now, although frank dissociative symptoms where awareness of time is lost (flashbacks) are less common in children. Because the re-experiencing symptoms are so distressing, children with PTSD develop overt or covert avoidance strategies, such as keeping themselves busy or distracted or staying away from people or places that remind them of the traumatic event. Alternatively, they may become upset if they find themselves in contexts resembling the traumatic event. Although avoidance might offer short term relief from distress, it does not reduce the occurrence of re-experiencing symptoms in the long term and may reduce engagement in enjoyable activities. Because of the often chronic, unpredictable nature of the distressing re-experiencing symptoms, children may feel under continued threat and therefore display physiological hyperarousal as they remain alert and vigilant for danger. Because of the hyperarousal, children with PTSD are often irritable, struggle to concentrate on daily tasks, and have problems sleeping.

On average, one in four trauma-exposed children develop PTSD by age 18 years, with a lifetime prevalence of 4.7-7.8\% in the general population. ${ }^{34}$ The prevalence of PTSD can be much higher in displaced children and those exposed to armed conflict. ${ }^{15}$ The risk of PTSD in individual children exposed to trauma depends on many characteristics including, importantly, the nature of the trauma. Traumas involving direct experiences of interpersonal violence, such as physical or sexual abuse, are most strongly associated with psychopathology. Children with PTSD are at high risk of harming themselves and of harming others because of aggression and risk taking. The odds of self harm are eight times higher in children with PTSD compared with children without PTSD, and the odds of suicide attempt are 10 times higher. In absolute terms, about half of children with PTSD report self harming and one in five report attempting suicide. The odds of violent offence records are also three times higher in children 
with PTSD compared with children without PTSD, corresponding to one in 10 children with PTSD. Finally, children with PTSD have significant functional impairment. They are three times more likely to be not in education, employment, or training (NEET) compared with children without PTSD, corresponding to one in four children with PTSD.

Although PTSD is a common clinical concern, the most prevalent disorders in children exposed to trauma are those also most prevalent in the general population. ${ }^{46}$ For example, while the 12 month prevalence of PTSD in trauma-exposed children is $14 \%$, the prevalence of depression is nearly $30 \%$, conduct disorder $23 \%$, and alcohol dependence $16 \%$. Of note, a PTSD diagnosis further increases the risk of comorbid psychiatric diagnoses, and more than three out of four children with PTSD have additional diagnoses ranging from internalising (such as depression or generalised anxiety) to externalising problems (such as conduct problems, substance misuse, or attention deficit and hyperactivity disorder) and psychotic symptoms. Beyond psychopathology, trauma-exposed children, on average, show small but pervasive cognitive deficits ${ }^{7}$ that may impair daily functioning and treatment response.

Although the association between child trauma and psychopathology is well established and likely causal, ${ }^{8}$ the mechanisms explaining this association are the focus of much current research. The traditional view has been that exposure to child trauma triggers a biological "toxic stress response" that alters brain function and ultimately contributes to psychopathology. ${ }^{9}$ Recent findings suggest that psychopathology is more strongly associated with the retrospective recall of childhood trauma (that is, subjective experience) than the actual exposure, pointing to psychological mechanisms involving biases in memory, core beliefs, and decision making. ${ }^{10}$ In contrast, the association between child trauma and cognitive deficits likely emerges from non-causal mechanisms and particularly pre-existing and stable differences in cognitive abilities that are risk factors for exposure to some trauma types. ${ }^{7}$ Finally, the complex clinical picture likely varies based on particular profiles of risk exposure in individual children, which can include both threats and deprivation (such as neglect or poverty),${ }^{11}$ highlighting the importance of dimensional models of risk measurement.

Because of the high prevalence and complexity of psychopathology related to child trauma, it is important to boost specialist training and clinical capacity in this area, which are often lacking in the community. Inadequate investment in this area has important negative consequences. One consequence is that, because of the complexity of clinical presentations, misdiagnosis or underdiagnosis is likely, leading to ineffective treatment and overload of other public services, such as the criminal justice system. ${ }^{.16}$ Another consequence is that, although natural recovery occurs in some cases, many children do not improve without treatment. ${ }^{17}$ Therefore, without timely evidence based treatment, children may go on to develop chronic or recurrent psychiatric disorders that become increasingly difficult to treat ${ }^{18}$ - with substantial individual and societal costs across the person's life course.

\section{Four ways to improve detection}

It is impossible to implement interventions if child trauma and trauma related psychopathology go undetected. In a recent population based British study, only $20 \%$ of trauma-exposed children sought help from general practitioners or mental health practitioners, and only $10 \%$ accessed mental health services in the past year. ${ }^{4}$ Rates of healthcare use are similarly low for children with trauma-related psychopathology. In the same cohort, only
$40 \%$ of children with PTSD sought help from GPs or mental health practitioners and only $20 \%$ accessed mental health services in the past year ${ }^{3}$ and might still be misdiagnosed or inadequately treated because of the lack of training in trauma-related disorders. Rates of healthcare use are almost certainly even lower in countries without universal healthcare or affected by conflict, emergencies, and disasters. There are at least four important steps to improving under-detection.

First, improve understanding of barriers to healthcare use. ${ }^{19}$ Some relate to the children, such as trauma-related psychological symptoms (for example, avoidance, low motivation, hopelessness, distrust); trauma-related cognitions (such as shame or guilt); or fear of reprisal by perpetrators or disbelief by family and adults. Other barriers relate to parents or care givers, such as deliberate concealment of trauma by perpetrators; fears of being blamed, having their child taken by child protective services, reprisal towards them or their child by perpetrators; poor mental health literacy; stigma around mental illness; and structural barriers (such as lack of insurance, access, or transportation).

Second, reduce these barriers by screening for trauma exposure. Screening is the first and necessary step to identify children who may develop trauma-related psychopathology. ${ }^{20}$ It can be undertaken in primary care or non-specialist health settings without significant distress for the children interviewed ${ }^{21}$ with short questionnaires (such as the Child Trauma Screen). However, like all screening programmes, it can only be ethically justified when it is implemented along with procedures to review the results of the screening and to provide further assessment and treatment to children who screen positive. ${ }^{22}$

Third, develop accurate prediction models to identify which trauma-exposed children are at greatest risk of developing psychopathology. Pragmatically, risk prediction is generally based on identification of early emotional and behavioural symptoms through screening instruments (such as the Child Revised Impact of Events Scale (CRIES), the Revised Children's Anxiety and Depression Scale (RCADS), and the Strengths and Difficulties Questionnaire (SDQ)). However, to enable interventions from the earliest stages in non-symptomatic trauma-exposed children and at scale, there is growing interest in developing individualised risk prediction models using modern statistical and machine learning methods. 423

Fourth, and more broadly, improve general practitioners' and primary care practitioners' training in child and adolescent mental health. Improved knowledge in this area can promote detection of psychological problems in children and referral to specialist mental health services.

\section{Evidence based treatment}

Evidence based psychological treatments for PTSD in children ${ }^{12-14}$ endorsed by guidelines from the National Institute for Health and Care Excellence (NICE) and the American Academy of Child and Adolescent Psychiatry include trauma-focused psychotherapies (such as trauma-focused cognitive behavioural therapy, cognitive therapy for PTSD, prolonged exposure, and narrative exposure therapy). Trauma-focused psychotherapies target cognitive and behavioural factors that contribute to the maintenance of PTSD and generally include psycho-education to provide information to children and families about PTSD symptoms and the treatment rationale; coping skills training to better manage intense negative emotions; gradual exposure to trauma memories and reminders to address avoidance and build a coherent trauma narrative; and cognitive restructuring to address biased appraisals related to the 
trauma memory. All individual forms of trauma-focused psychotherapies have large effects in reducing PTSD symptoms compared with waiting list ${ }^{24}$ and are cost effective in the treatment of PTSD in children. ${ }^{25}$

Limited but growing evidence indicates that eye movement desensitisation and reprocessing (EMDR), ${ }^{12-14}$ which involves recalling traumatic events while performing tasks that generate bilateral sensory stimulation, may be beneficial for young people with PTSD. Pharmacological interventions are not effective for treating PTSD in young people ${ }^{12-14}$ but can be effective for treatment of comorbid disorders (such as depression and anxiety).

Because PTSD is not the only or the most common diagnosis in trauma-exposed children, ${ }^{4}{ }^{6}$ treatment recommendations for each trauma-exposed child should be guided by the diagnoses made by experienced clinicians. It is important to consider how trauma may organise and provide distinctive features to the clinical presentation. Because psychiatric comorbidity ${ }^{4}$ is common, trans-diagnostic approaches that target common mechanisms underlying the co-occurrence of different disorders are gaining popularity. ${ }^{26}$

There is also much interest in early interventions to reduce psychopathology in trauma-exposed children, because of the identifiable external cause. As in adults, universal single-session debriefing is not beneficial for trauma-exposed children. ${ }^{13}{ }^{14}$ More targeted interventions have yielded better results, however. For example, children who reported at least one new PTSD symptom after trauma exposure and received a family intervention targeting psycho-education about trauma symptoms, improved family communication, and teaching of coping skills to manage symptoms were significantly less likely to have a PTSD diagnosis three months after the intervention. ${ }^{27}$ Furthermore, focused psychosocial interventions can be effective for children in low-resource humanitarian settings ${ }^{28} 29$ or among refugees, asylum seekers, or internally displaced people. ${ }^{30}$

\section{Key recommendations}

- To improve detection of childhood trauma-related psychopathology, it is important to better understand barriers to healthcare use, implement screening procedures, develop accurate prediction models, and improve knowledge of child and adolescent mental health problems among primary care practitioners

- To improve treatment of childhood trauma-related psychopathology, more specialist training and clinical capacity in this subject is needed for child and adolescent mental health practitioners

Contributors: AD wrote an initial draft manuscript, contributed to critical revisions to the text, and is the guarantor of this article. KAM, MS, and CSS contributed to critical revisions of the text. All authors have been engaged in research and clinical practice with trauma-exposed children for more than a decade.

Funding: AD was funded by the Medical Research Council (grant No P005918) and the National Institute for Health Research (NIHR) Biomedical Research Centre at South London and Maudsley NHS Foundation Trust and King's College London. KAM was funded by the National Institute of Mental Health (NIMH; R01-MH103291, R01-MH106482, R56-MH119194, R37-MH119194). MS was funded by Qatar National Research Fund (QNRF grant No NPRP 7-154-3-034). The views expressed are those of the authors and not necessarily those of the funders.

Competing interests: We have read and understood BMJ policy on declaration of interests and have no relevant interests to declare.

Provenance and peer review: Commissioned; externally peer reviewed.

This article is part of a series commissioned by The BMI for the World Innovation Summit for Health (WISH) 2020. The BM/peer reviewed, edited, and made the decisions to publish. The series, including open access fees, is funded by WISH.

American Psychiatric Association. Diagnostic and statistical manual of mental disorders. 5th ed. APA, 2013.
2 Nelson CA, Scott RD, Bhutta ZA, etal. Adversity in childhood is linked to mental and physical health throughout life. BMJ2020;370:m3048. doi: 10.1136/bmj.m3048.

McLaughlin KA, Koenen KC, Hill ED, etal. Trauma exposure and posttraumatic stress disorder in a national sample of adolescents. J Am Acad Child Adolesc Psychiatry 2013;52:815-830.e14. doi: 10.1016/j.jaac.2013.05.011. pmid: 23880492

4 Lewis SJ, Arseneault L, Caspi A, etal. The epidemiology of trauma and post-traumatic stress disorder in a representative cohort of young people in England and Wales. Lancet Psychiatry 2019;6:247-56. doi: 10.1016/S2215-0366(19)30031-8. pmid: 30798897

5 Kohrt BA, Jordans M), Tol WA, etal. Comparison of mental health between former child soldiers and children never conscripted by armed groups in Nepal. JAMA 2008;300:691-702 doi: 10.1001/jama.300.6.691. pmid: 18698067

6 Copeland WE, Keeler G, Angold A, Costello EJ. Traumatic events and posttraumatic stress in childhood. Arch Gen Psychiatry2007;64:577-84. doi: 10.1001/archpsyc.64.5.577. pmid: 17485609

7 Danese A, Moffitt TE, Arseneault L, etal. The origins of cognitive deficits in victimized children: implications for neuroscientists and clinicians. Am J Psychiatry 2017:174:349-61. doi: 10.1176/appi.ajp.2016.16030333. pmid: 27794691

8 Schaefer JD, Moffitt TE, Arseneault L, etal. Adolescent victimization and early-adult psychopathology: approaching causal inference using a longitudinal twin study to rule out noncausal explanations. Clin Psychol Sci 2018;6:352-71. doi: 10.1177/2167702617741381. pmid: 29805917

9 Danese A, McEwen BS. Adverse childhood experiences, allostasis, allostatic load, and age-related disease. Physiol Behav 2012;106:29-39. doi: 10.1016/.jphysbeh.2011.08.019. pmid: 21888923

10 Danese A, Widom CS. Objective and subjective experiences of child maltreatment and their relationships with psychopathology. Nat Hum Behav 2020; doi: 10.1038/s41562-020-0880-3. pmid: 32424258

11 McLaughlin KA, Sheridan MA. Beyond cumulative risk: a dimensional approach to childhood adversity. Curr Dir Psychol Sci2016;25:239-45. doi: 10.1177/0963721416655883. pmid: 27773969

12 Cohen JA, Bukstein O, Walter H, etalAACAP Work Group On Quality Issues. Practice parameter for the assessment and treatment of children and adolescents with posttraumatic stress disorder J Am Acad Child Adolesc Psychiatry 2010;49:414-30. doi: 10.1016/j.jaac.2009.12.020. pmid: 20410735

13 National Institute for Health and Care Excellence. Post-traumatic stress disorder (NICE guideline NG116). 2018. https://www.nice.org.uk/guidance/ng116

14 Smith P, Dalgleish T, Meiser-Stedman R. Practitioner Review: Posttraumatic stress disorder and its treatment in children and adolescents. J Child Psychol Psychiatry 2019;60:500-15. doi: 10.1111/jcpp.12983. pmid: 30350312

15 Samara M, Hammuda S, Vostanis P, El-Khodary B, Al-Dewik N. Rethinking trauma and resilience in the context of political violence: New directions in research and practice. BM/2020;370:m3155 doi: 10.1136/bmj.m3155.

16 Abram KM, Teplin LA, Charles DR, Longworth SL, McClelland GM, Dulcan MK. Posttraumatic stress disorder and trauma in youth in juvenile detention. Arch Gen Psychiatry2004;61:403-10 doi: 10.1001/archpsyc.61.4.403. pmid: 15066899

17 Hiller RM, Meiser-Stedman R, Fearon P, etal. Research review: Changes in the prevalence and symptom severity of child post-traumatic stress disorder in the year following trauma - a meta-analytic study. J Child Psychol Psychiatry 2016;57:884-98. doi: 10.1111/jcpp.12566. pmid: 27169987

18 Nanni V, Uher R, Danese A. Childhood maltreatment predicts unfavorable course of illness and treatment outcome in depression: a meta-analysis. Am J Psychiatry 2012;169:141-51. doi: 10.1176/appi.ajp.2011.11020335. pmid: 22420036

19 Gulliver A, Griffiths KM, Christensen H. Perceived barriers and facilitators to mental health help-seeking in young people: a systematic review. BMC Psychiatry 2010;10:113. doi: 10.1186/1471-244X-10-113. pmid: 21192795

20 Bhushan D, Argueza BR, Machtinger E, Harris NB. Screening and intervening on adverse childhood experiences (ACEs) and toxic stress. BMJ [forthcoming].

21 Finkelhor D, Vanderminden J, Turner H, Hamby S, Shattuck A. Upset among youth in response to questions about exposure to violence, sexual assault and family maltreatment. Child Abuse Neg/ 2014;38:217-23. doi: 10.1016/j.chiabu.2013.07.021. pmid: 24004683

22 Dubowitz H, Feigelman S, Lane W, Kim J. Pediatric primary care to help prevent child maltreatment the Safe Environment for Every Kid (SEEK) Model. Pediatrics 2009;123:858-64. doi: 10.1542/peds.2008-1376. pmid: 19255014

23 Meehan AJ, Latham RM, Arseneault L, Stahl D, Fisher HL, Danese A. Developing an individualized risk calculator for psychopathology among young people victimized during childhood: A population-representative cohort study. J Affect Disord 2020;262:90-8. doi: 10.1016/j.jad.2019.10.034. pmid: 31715391

24 Mavranezouli I, Megnin-Viggars O, Daly C, etal. Research review: Psychological and psychosocial treatments for children and young people with post-traumatic stress disorder: a network meta-analysis. J Child Psychol Psychiatry2020;61:18-29. doi: 10.1111/jcpp.13094. pmid: 31313834

25 Mavranezouli I, Megnin-Viggars O, Trickey D, etal. Cost-effectiveness of psychological interventions for children and young people with post-traumatic stress disorder. J Child Psychol Psychiatry 2020;61:699-710. doi: 10.1111/jcpp.13142. pmid: 31654414

26 McLaughlin KA, Colich NL, Rodman AM, Weissman DG. Mechanisms linking childhood trauma exposure and psychopathology: a transdiagnostic model of risk and resilience. BMC Med 2020;18:96. doi: 10.1186/s12916-020-01561-6. pmid: 32238167 


\section{ANALYSIS}

27 Berkowitz SJ, Stover CS, Marans SR. The Child and Family Traumatic Stress Intervention: secondary prevention for youth at risk of developing PTSD. I Child Psychol Psychiatry 2011;52:676-85. doi: 10.1111/j.1469-7610.2010.02321.x. pmid: 20868370

28 Purgato M, Gross AL, Betancourt T, etal. Focused psychosocial interventions for children in low-resource humanitarian settings: a systematic review and individual participant data meta-analysis. Lancet Glob Health 2018;6:e390-400. doi: 10.1016/S2214-109X(18)30046-9. pmid: 29530422

29 El-Khodary B, Samara M. Effectiveness of a school-based intervention on the students' mental health after exposure to war-related trauma. Front Psychiatry 2020;10:1031. doi: 10.3389/fpsyt.2019.01031. pmid: 32273852

30 Samara M, El Asam A, Khadaroo A, Hammuda S. Examining the psychological well-being of refugee children and the role of friendship and bullying. Br J Educ Psychol 2020;90:301-29. do: 10.1111/bjep.12282. pmid: 31056751

This is an Open Access article distributed in accordance with the Creative Commons Attribution Non Commercial (CC BY-NC 4.0) license, which permits others to distribute, remix, adapt, build upon this work non-commercially, and license their derivative works on different terms, provided the original work is properly cited and the use is non-commercial. See: http://creativecommons.org/icenses/by$\mathrm{nc} / 4.0 \%$ 